Enfermagem Brasil 2016;15(5):151-8

\title{
ARTIGO ORIGINAL \\ Avaliação do consumo alimentar em residências de crianças usuárias de um centro de educação infantil
}

Revillyn Souza da Silva*, Adriana Cecel Guedes, M.Sc.**, Bruna Gabriela Bibancos Damas, M.Sc. ***

${ }^{*}$ Enfermeira, Universidade Paulista, ${ }^{* *}$ Coordenadora do Curso de Enfermagem do Campus Tatuapé/SP da Universidade Paulista, ${ }^{* *}$ Docente na Universidade Paulista

Recebido em 30 de agosto de 2016; aceito em 7 de outubro de 2016.

Endereço para correspondência: Revillyn Souza da Silva, Rua João Pinho 174, Jd. Vila Carrão São Mateus 08340-380 São paulo SP, E-mail: revillyn@hotmail.com; Adriana Cecel Guedes: adricecel@gmail.com, Bruna Gabriela Bibancos Damas: brudamas1@hotmail.com

\section{Resumo}

É fundamental para um crescimento adequado e saudável da criança uma alimentação adequada em casa. O objetivo deste estudo foi avaliar a alimentação consumida na residência de crianças usuárias de um centro de educação infantil, em quantidade e qualidade. Trata-se de uma pesquisa de campo descritiva exploratória com abordagem quantitativa, realizada em um Centro de Educação Infantil (CEI) da cidade de São Paulo. Participaram deste estudo 57 mães de crianças usuárias do CEI; $53 \%$ das crianças eram do sexo feminino e $47 \%$ do sexo masculino; $75 \%$ das crianças foram classificadas com o peso ideal; todas as crianças foram amamentadas. A maior parte das crianças frequentava restaurantes do tipo fast food, consumia produtos industrializados de diferentes tipos e ingeria bebidas industrializadas e naturais, mas com alta frequência de consumo de suco industrializado. Nem todas consumiam água em casa. Neste estudo concluiu-se que apesar de toda uma dieta adequada no $\mathrm{CEI}$, os hábitos alimentares da família faz com que a criança consuma alimentos industrializados que podem afetar à saúde. Entretanto, percebeu-se também a ingestão de verduras, legumes e frutas com frequência e a maioria estava no peso ideal para a idade.

Palavras-chave: alimentação infantil, estado nutricional, alimentos industrializados.

\section{Abstract \\ Evaluation of nutritional intake at home of children attended at an educational center}

According to studies, in the first years of life children should eat appropriate and healthy meal at home. The objective of this study was to evaluate the nutritional intake, at home, of children attending a children educational center, in quantity and quality. This was an exploratory descriptive field research with quantitative approach, performed at a Children's Educational Center (CEC) in São Paulo city. Fifty seven mothers of children attending the CEC were enrolled in this study; $53 \%$ of children were female, and was male; $75 \%$ of children were classified as normal weight; and all children were breastfed. Most of these children go to fast food restaurants and consume different kinds of processed food. These children intake industrialized and natural beverages, with high prevalence intake of industrialized juices. Not all children drink water at home. The study concluded that children had an adequate diet at CEC, however the eating habits of their family make the children consume processed foods, which may affect their health. Meanwhile, children consumed vegetables and fruits, and most of them were classified as normal weight.

Key-words: child nutrition, nutritional status, processed foods.

\section{Resumen \\ Evaluación del consumo alimentario en el domicilio de niños que frecuentan un Centro de Enseñanza Infantil}

Es fundamental para el desarrollo adecuado y saludable del niño una alimentación adecuada en casa. El objetivo de este estudio ha sido evaluar la alimentación de niños que frecuentan un centro de enseñanza infantil, consumida en su domicilio, en cantidad y calidad. Se trata de una investigación descriptiva exploratoria con enfoque cuantitativo con investigación de campo, realizada en Centro de Enseñanza Infantil (CEI) en la ciudad de São Paulo. Participaron de este estudio 57 madres de niños que frecuentaban el CEl: el $53 \%$ eran niñas y el $47 \%$ niños. El 
$75 \%$ de los niños fueron considerados con peso normal y todos fueron amamantados. La mayor parte de los niños frecuentaba restaurant fast food (comida rápida) y consumía productos industrializados de diferentes tipos; ingerían bebidas industrializadas y naturales, con alta frecuencia de consumo de jugo industrializado; y no todos bebían agua en sus casas. Se concluye que aunque la dieta en el CEI sea adecuada, los hábitos alimentarios de la familia hacen con que los niños consuman productos industrializados que pueden afectar la salud. No obstante, se percibió también la ingesta de verduras, frutas y legumbres con frecuencia y la mayoría tenía un peso normal.

Palabras-clave: nutrición del niño, estado nutricional, alimentos industrializados.

Introdução

Uma alimentação adequada é fundamental para a promoção da saúde e deve atender às necessidades nutricionais da criança em cada faixa etária proporcionando seu crescimento e desenvolvimento saudável e harmoniosos [1]. Os hábitos alimentares são adquiridos durante toda a vida, destacando-se os primeiros anos como um período de grande importância para o estabelecimento de hábitos que promovam a saúde do indivíduo [2].

As instituições de educação infantil têm a responsabilidade em oferecer alimentos de qualidade nutricional e incentivar às crianças a consumir diferentes tipos de alimentos, este incentivo deve ser enfatizado tanto no ambiente escolar quanto no domicílio [3]. Os centros de educação infantil é um lugar onde as crianças permanecem a maior parte do tempo, a rotina alimentar é realizada por nutricionistas, respeitando as fases de desenvolvimento da criança, assim como o preparo dos alimentos recebe uma atenção especial. A alimentação deve ser estimulante para a criança, pois está diretamente ligada à formação dos hábitos alimentares, bem como a escolha correta dos alimentos [1]. No CEI a participação da criança nas atividades relacionadas à alimentação deve ser estimulada, seus interesses alimentares, habilidades e desenvolvimento devem ser respeitados [1].

A família é responsável pela formação do hábito alimentar da criança e do seu comportamento alimentar, que se forma através da aprendizagem social, desempenhando os pais o papel de primeiros educadores nutricionais. Os fatores psicossociais e culturais influenciam as experiências alimentares da criança, desde o momento de nascimento, dando início ao processo de aprendizagem [4].

Diante disso, para que a alimentação tenha qualidade é necessário esforço conjunto da CEI e da família. Assim, este estudo teve como questionamento: Como é a alimentação da criança fora da CEI?

\section{Material e métodos}

Este estudo tem caráter descritivo exploratório com abordagem quantitativa, com pesquisa de campo.

A pesquisa foi realizada no Centro de Educação Infantil localizada na zona leste de São Paulo. Dentro dessa CEI ocorre um trabalho de promoção da saúde da criança, realizado pela clínica de saúde da UNIP desde 2012.

A população do estudo foram os 74 alunos matriculados na CEI, destes 57 alunos com idade entre 1 ano a 4 anos aceitaram participar da pesquisa.

Foram considerados critérios de inclusão: a) Ser pai ou familiar da criança usuário da CEI; b) Concordância em participar voluntariamente da pesquisa; c) Assinar o Termo de Consentimento Livre e Esclarecido (TCLE) para menores;

Foram considerados critérios de exclusão: a) Não ser pai ou familiar da criança usuária do CEl; b) Relutância em participar voluntariamente da pesquisa. c) Não assinar o TCLE.

Os dados foram coletados através de um questionário composto por questões objetivas, acerca dos dados demográficos e alimentação da criança em casa. As questões relacionadas ao peso e altura da criança foram coletadas de prontuários de saúde dos alunos, vindo do trabalho de promoção da saúde realizado pela UNIP, os questionários foram passados entre os meses de dezembro a março de 2015.

O questionário foi entregue pela diretora da CEI as mães, quando foi explicada a pesquisa. Os questionários foram entregues com o TCLE para menores de idade, deixou claro que a participação era voluntária e todos poderiam recusar-se a participar da pesquisa a qualquer momento. 
As informações obtidas foram armazenadas no Microsoft Excel e analisadas de maneira descritiva, calculando as frequências absolutas e relativas para todas as variáveis. Os dados foram apresentados em forma de tabelas e discutidos com literatura especifica.

Foi feito o índice de massa corpórea IMC com base na proposta dos escores- $Z$ da caderneta da saúde da criança do Ministério da Saúde 2011, utilizando os gráficos recomendados para crianças de $0-5$ anos. Considerando os índices IMC/I foram adotados os pontos de corte $>+3$ escores $z$ para identificar crianças com obesidade, $\leq+3$ e $\geq+2$ escores $z$ para identificar sobrepeso, $\leq+2 \mathrm{e} \geq+1$ escores $z$ para identificar risco de sobrepeso, $\leq+1$ e $\geq$ 2 escores $z$ para identificar IMC adequado, $\leq 2$ e $\geq-3$ escores $z$ para identificar magreza $e<-3$ escores z para identificar magreza acentuada [5].

Dado que os sujeitos do estudo são seres humanos, obedecemos ao previsto na Resolução 466/12 no Ministério da Saúde, submetendo a análise e julgamento do Comitê de Ética em pesquisa com seres humanos da UNIP que é reconhecido pelo Conselho Nacional de Pesquisa com seres humanos (CONEP). A pesquisa foi aprovada pelo Comitê de Ética no dia 14/08/2014 com o número do parecer 755.211.

Resultados

Fizeram parte deste estudo 57 mães de crianças usuárias do Centro de Educação Infantil (CEI) da Cidade de São Paulo. O número total de crianças matriculadas no CEI era de 74 crianças, foram excluídas do estudo 14 crianças cujos pais não aceitaram participar da pesquisa. Dessas crianças, $53 \%$ eram do sexo feminino e $47 \%$ do sexo masculino. Quanto à idade, $23 \%$ tinham 1 ano, 30\%, 2 anos, 37\%, 3 anos, e 10\%, 4 anos de idade.

Todas as crianças foram amamentadas. Dezoito por cento (18\%) foram amamentadas apenas durante 6 meses, $65 \%$ foram amamentadas durante o período de 7 a 12 meses, $9 \%$ interromperam a amamentação após a criança completar 1 ano, e 3\% amamentaram até 2 anos.

Em relação ao peso e altura, foi feito o IMC de $82 \%$ das crianças, não participaram desta fase $18 \%$, por não terem os dados no prontuário de saúde da criança. Seguindo o IMC proposto pela organização mundial da Saúde (OMS) para crianças, $72 \%$ foram classificadas como peso normal, $5 \%$ em risco de sobrepeso, e $5 \%$ obesidade.

A média do peso dos meninos de 1 e 2 anos foi de 13,04, DP $\pm 5,7$, a média do comprimento foi de 82,77, DP $\pm 32,24$. A média de peso dos meninos de 3 e 4 anos foi de 13,96 , DP $\pm 5,61$, a média de comprimento foi de 86,53, DP $\pm 30,90$. Já nas meninas de 1 e 2 anos foi de 12,63 , DP $\pm 5,89$, a média de comprimento foi de $80,70, \mathrm{DP} \pm 33,63$. Nas meninas de 3 e 4 anos a média foi de 13,99, DP $\pm 5,45$, a média do comprimento foi de 86,96 , DP \pm 30,13 .

A tabela I descreve a frequência que a criança vai a um restaurante do tipo fast food, alimentos solicitados com maior frequência por ela, e frequência de consumo desses alimentos. A maior parte das crianças frequentava restaurantes do tipo fast food e consumía produtos industrializados de diferentes tipos. O número de alimentos industrializados consumidos é maior que 57, porque alguns pais responderam que a criança consome mais de um tipo de alimento. Ou seja, esses tipos de alimentos fazem parte da dieta das crianças. 
Tabela I - Frequência que a criança do CEI vai a um restaurante do tipo fast food, alimentos solicitados com maior frequência pela criança e frequência de consumo desses alimentos. São Paulo, 2015.

\begin{tabular}{lll}
\hline Frequência do consumo de fast food & $\mathbf{f}$ & $\%$ \\
\hline 1 vez na semana & 5 & 8 \\
Mais de 1 vez na semana & 1 & 2 \\
1 vez ao mês & 50 & 88 \\
Não frequenta & 1 & 2 \\
Total & 57 & 100 \\
& $\mathbf{f}$ & $\%$ \\
Pizza & 13 & 8 \\
Achocolatado & 37 & 22 \\
Hambúrguer & 23 & 14 \\
Salgadinhos & 57 & 35 \\
Chocolate/Bombom & 34 & 21 \\
Total & 164 & 100 \\
& $\mathbf{f}$ & $\%$ \\
1 vez ao dia & 13 & 23 \\
2 vezes ao dia & 37 & 65 \\
3 vezes ao dia & 7 & 12 \\
Mais de 3 vezes ao dia & 0 & 0 \\
Não consumo & 0 & 0 \\
Total & 57 & 100 \\
\hline & &
\end{tabular}

As crianças consumiam bebidas industrializadas e naturais de diferentes tipos com alta frequência de consumo do suco industrializado $88 \%$, água $74 \%$, suco natural de frutas $58 \%$ e por último o refrigerante $49 \%$. A tabela II descreve o consumo de refrigerante, suco industrial por dia.

Tabela II - Frequência de consumo ao dia de suco industrializado e refrigerante pela criança do CEl quando está em casa. São Paulo, 2015.

\begin{tabular}{lll}
\hline Frequência & $\mathbf{f}$ & $\%$ \\
1 vez ao dia & 30 & 53 \\
2 vezes ao dia & 22 & 39 \\
3 vezes ao dia & 2 & 3 \\
Mais de 3 vezes ao dia & 0 & 0 \\
Não consome & 3 & 5 \\
Total & 57 & 100 \\
\hline
\end{tabular}

A tabela III descreve a frequência de consumo ao dia na alimentação da criança, por classe dos alimentos: cereais, verduras e legumes, frutas, leites e derivados, feijões, gorduras, açúcar e doces e carnes e ovos.

Em relação à idade; $89,4 \%$ das crianças de 1 a 3 anos consumiam cereais com maior frequência de consumo 2 vezes ao dia $(76,4 \%)$, quando estava em casa; verduras e legumes, 2 vezes ao dia (59\%); frutas, 2 vezes ao dia (53\%); na classe do leite, queijo e iogurte, a frequência de consumo ao dia foi 3 vezes ao dia (59\%); carnes e ovos, 2 vezes ao dia $(82,3 \%)$; feijão, 2 vezes ao dia (70,5\%); gorduras, 2 vezes ao dia (67\%); e a classe do açúcar e doces, também 2 vezes ao dia $(72,5 \%)$.

Já nas crianças de 4 anos $(10,5 \%)$, a maior frequência de consumo do grupo dos cereais foi de 2 vezes ao dia (100\%); no grupo das verduras e legumes a frequência de consumo ao dia foi dividida, $50 \%$ consumiam 1 vez ao dia e $50 \%$ consumiam 2 vezes ao dia; na classe das frutas a maior frequência foi 3 vezes ao dia (67\%); Leite, queijo e iogurte, 2 vezes ao dia (67\%); carnes e ovos, 2 vezes ao dia (100\%); do grupo do feijão, 2 vezes ao dia $(83,3 \%)$; da classe da gordura, 1 vez (50\%); e na classe do açúcar e doces, 2 vezes (100\%). 
Tabela III - Distribuição da frequência de consumo diário por classe dos alimentos pela criança do CEI $(n=57)$. São Paulo, 2015.

\begin{tabular}{|c|c|c|c|c|c|}
\hline Cereais & $f$ & $\%$ & Feijões & $f$ & $\%$ \\
\hline 1 vez ao dia & 2 & 3 & 1 vez ao dia & 14 & 25 \\
\hline 2 vezes ao dia & 45 & 79 & 2 vezes ao dia & 41 & 72 \\
\hline 3 vezes ao dia & 9 & 16 & 3 vezes ao dia & 2 & 3 \\
\hline Mais de 3 vezes ao dia & 0 & 0 & Mais de 3 vezes ao dia & 0 & 0 \\
\hline Não consome & 1 & 0 & Não consome & 0 & 0 \\
\hline Total & 57 & 2 & Total & 57 & 100 \\
\hline Verduras e legumes & f & $\%$ & Gorduras & f & $\%$ \\
\hline $1 \mathrm{vez}$ ao dia & 19 & 33 & 1 vez ao dia & 9 & 16 \\
\hline 2 vezes ao dia & 33 & 59 & 2 vezes ao dia & 36 & 63 \\
\hline 3 vezes ao dia & 3 & 5 & 3 vezes ao dia & 9 & 16 \\
\hline Mais de 3 vezes ao dia & 2 & 3 & Mais de 3 vezes ao dia & 2 & 3 \\
\hline Não consome & 0 & 0 & Não consome & 1 & 2 \\
\hline Total & 57 & 100 & Total & 57 & 100 \\
\hline Frutas & f & $\%$ & Açúcar, doces & f & $\%$ \\
\hline 1 vez ao dia & 2 & 3 & 1 vez ao dia & 5 & 9 \\
\hline 2 vezes ao dia & 29 & 51 & 2 vezes ao dia & 43 & 75 \\
\hline 3 vezes ao dia & 25 & 44 & 3 vezes ao dia & 8 & 14 \\
\hline Mais de 3 vezes ao dia & 1 & 2 & Mais de 3 vezes ao dia & 1 & 2 \\
\hline Não consome & 0 & 0 & Não consome & 0 & 0 \\
\hline Total & 57 & 100 & Total & 57 & 100 \\
\hline Leites, queijos e Danoninho & f & $\%$ & Carne e ovos & f & $\%$ \\
\hline $1 \mathrm{vez}$ ao dia & 3 & 5 & 1 vez ao dia & 4 & 8 \\
\hline 2 vezes ao dia & 22 & 39 & 2 vezes ao dia & 48 & 84 \\
\hline 3 vezes ao dia & 32 & 56 & 3 vezes ao dia & 2 & 3 \\
\hline Mais de 3 vezes ao dia & 0 & 0 & Mais de 3 vezes ao dia & 3 & 5 \\
\hline Não consome & 0 & 0 & Não consome & 0 & 0 \\
\hline Total & 57 & 100 & Total & 57 & 100 \\
\hline
\end{tabular}

Discussão

As crianças no CEI são divididas em grupos conforme a idade, os grupos são: berçário, minigrupo I e minigrupo II, a maioria das crianças era do sexo feminino $53 \%$ e tinham 3 anos de idade (37\%). Estudo realizado no município de São Paulo com 106 crianças também teve a maior prevalência de crianças do sexo feminino e a maioria das crianças tinha mais de 1 ano de idade [6].

Observou-se que a maioria das crianças (65\%) foram amamentadas por mais de 6 meses, e apenas 3\% foram amamentadas até 2 anos. A Organização Mundial da Saúde preconiza que a criança seja amamentada exclusivamente até 6 meses e, a partir daí, entrar com alimentos complementares na dieta da criança, mas mantendo a amamentação até 2 anos [7]. O leite materno é muito importante para a mãe e o bebê por vários motivos, entre eles melhorar a recuperação pós-parto e proteger o bebê contra infecções [8].

Estudo realizado no Município de Patos/PB obteve que a maioria $(40 \%)$ das mães amamentaram seus filhos por um período de 6 a 12 meses [9]. Neste estudo, $65 \%$ das crianças foram amamentadas por um período maior de 7 aos 12 meses. Outro estudo realizado em Fortaleza com 20 mães de crianças menores de 6 meses notou que um dos motivos do desmame precoce foi o trabalho fora de casa da mãe [10].

Sobre o valor da relação entre índice de massa corpórea e idade (IMC/I), não foi identificada nenhuma criança com risco de desnutrição ou com desnutrição. Setenta e dois por cento $(72 \%)$ das crianças foram classificadas como peso normal para a idade, $5 \%$ das crianças encontravam-se em risco de sobrepeso e $5 \%$ obesas. Um estudo, realizado no município de São Paulo (SP) com 66 pré-escolares de 2 a 6 anos de idade, verificou que 3,9\% dos préescolares de 2 a 3 anos e $5 \%$ dos pré-escolares de 4 a 6 anos encontravam-se com baixo IMC/I; 6,4\% excesso de peso e 25,3\% encontravam-se em risco de sobrepeso [11]. 
Já outro estudo, realizado no município de Viçosa/MG com 89 crianças de 24 a 72 meses de idade, seguindo o escore-Z para o índice de peso/idade (PI), obteve um valor de $59,8 \%$ das crianças com peso ideal, $27,6 \%$ em risco de desnutrição e $6,9 \%$ em risco de obesidade [12]. Nesse estudo apareceram crianças em risco de desnutrição, o que difere deste estudo. Essa diferença pode estar ligada a fatores como perfil social dos pais, tipo de alimentação na creche ou orientações recebidas pelos pais.

Quanto ao consumo de alimentos industrializados, $88 \%$ das mães relataram que a criança consumia fast food pelo menos 1 vez ao mês e que os alimentos industrializados citados no questionário foram consumidos 2 vezes ao dia em $65 \%$ das crianças. A criança passava a maior parte do dia no $\mathrm{CEI}$, mesmo assim é alta a frequência de consumo de 2 vezes ao dia de alimentos industrializados quando está em casa, dentre os alimentos citados na tabela I o mais solicitado pela criança foi o salgadinho com $35 \%$.

O consumo de alimentos industrializados no curto tempo que a criança está em casa, pode acontecer pelo fato de os alimentos industrializados serem mais aceitos pelas crianças e ser mais fáceis de serem preparados pelos familiares. Isso também pode ser consequência do aumento do acesso desses alimentos a família, já que houve aumento da renda per capta da população [13], e pela influência de hábitos alimentares dos pais.

Estudo realizado representando um recorde da Pesquisa Nacional de Demografia e Saúde (PNDS) em 2006, sobre o consumo alimentar entre crianças brasileiras de dois a cinco anos de idade, o alimento não saudável consumido ao dia com maior frequência $47,4 \%$ foi biscoito ou bolacha [13]. Outro estudo realizado em creches do município de São Paulo observou que os alimentos industrializados são muito utilizados na alimentação infantil, por serem práticos e saborosos [14].

Apesar do consumo de alimentos industrializados em casa, a maioria das crianças ainda está no peso ideal, mas vale ressaltar que esse tipo de consumo merece atenção por parte dos pais, uma vez que outras alterações podem ocorrer como hipertensão arterial e dislipidemias.

Os alimentos industrializados não são alimentos adequados para a dieta da criança, devido à alta taxa de gorduras e açúcares e outros aditivos alimentares. Esses alimentos em excesso fazem mal a saúde, acarreta risco de doenças, principalmente a obesidade infantil. $O$ consumo dos mesmos deve ser desencorajado ou diminuído nos primeiros anos de vida, como forma de prevenção e promoção do desenvolvimento adequado [15].

Foi avaliado o consumo de líquidos em casa pela criança do CEI, destacou-se o maior consumo de suco do tipo néctar de fruta $88 \%$, entretanto vários líquidos são consumidos pela criança ao dia, quando está em casa. A frequência de consumo ao dia quando a criança está em casa de suco industrial e refrigerante foi de 2 vezes. $O$ consumo desse tipo de bebida merece cuidado, pode levar a redução do consumo de suco natural de frutas, e está associado a alergias alimentares [7]. É importante estimular a diminuição de consumo de refrigerante e suco industrial por ter aditivos que podem fazer mal a saúde [7].

Em relação ao consumo de água, $74 \%$ das crianças consomem água quando está em casa e $26 \%$ não a consomem, o que pode ser consequência do maior consumo de suco industrializado. Estudo realizado na Bahia com crianças menores de 1 ano mostrou que crianças não amamentadas consumiam mais água, chás e sucos. Esses tipos de bebidas não precisam ser ofertados a crianças amamentadas nos primeiros meses de vida, o leite tem tudo que ela precisa por isso a importância da amamentação [7-16].

Os guias alimentares trazem recomendações de uma dieta saudável, para cada faixa etária [17], assim analisou-se o consumo diário ao dia quando as crianças estão em casa, por classe dos alimentos.

A primeira classe analisada foi a dos cereais ao dia quando a criança está em casa, a frequência foi de 2 vezes ao dia (79\%). Segundo a Sociedade Brasileira de Pediatria (SBP), as porções indicadas para a faixa etária do estudo é de 5 porções ao dia. Os alimentos desta classe são fontes de carboidratos de energia muito importante para crianças que nessa faixa precisam de energia para brincar, por exemplo [18].

$\mathrm{Na}$ classe das verduras e legumes, a frequência de consumo no estudo foi de 2 vezes ao dia (59\%), quando comparado entre as porções recomendadas pela pirâmide alimentar infantil brasileira está abaixo do recomendado que é de 3 porções ao dia. Os alimentos desta classe são fontes de vitaminas e minerais [18]. Entretanto mais porções desses alimentos são consumidas na creche. Estudo realizado na Lituânia sobre o consumo alimentar em fase préescolar nos anos 2002, 2006 e 2010 resultou no alto consumo de doces, chocolates, biscoitos, bolos e baixo consumo de frutas e legumes [19]. 
Na classe das frutas, $89 \%$ das crianças de 1 e 3 anos e $11 \%$ de 4 anos consumiam 2 vezes ao dia. Segundo a pirâmide alimentar infantil, a porção recomendada para crianças de 1 a 3 anos são 4 porções e crianças de 4 anos 3 porções diárias. Para as porções diárias do leite, queijo e iogurte o consumo ao dia foi de 3 vezes (56\%), nesta classe foram atendidas as recomendações da pirâmide alimentar de 3 porções, adequado ao consumo das crianças [20].

$\mathrm{Na}$ classe da gordura, a recomendação da porção ao dia também é diferente conforme a faixa etária, o estudo mostrou um consumo de $67 \%$ das crianças de 1 a 3 anos de 2 vezes ao dia e de 4 anos (11\%) 1 vez ao dia (50\%). Nessa classe também foi atendida a recomendação pela pirâmide da porção recomendada [18-20]. Visto que porções desse tipo de alimento também são oferecidas à criança na creche, o total de consumo diário ultrapassa 0 recomendado.

Foi observada, na classe do açúcar e doces, uma frequência de consumo de 2 vezes ao dia (75\%), ultrapassando a porção recomendada que seja de 1 porção ao dia. Segundo a pirâmide alimentar infantil, é importante a redução dessa classe na alimentação das crianças em casa, evitando o risco de doenças [18-20].

E por fim, foi observada, no grupo das carnes e ovos, uma frequência de consumo ao dia em casa de 2 vezes ao dia (84\%) porções adequadas segundo a pirâmide de alimentação infantil que recomenda 2 porções de alimentos desta classe, já que são fontes de ferro, vitaminas e proteínas [20].

No estudo realizado em Viçosa/MG, quando avaliada a frequência de consumo dos grupos alimentares, foi identificado baixo consumo de frutas, hortaliças, raízes e tubérculos e carnes, e a maior frequência de consumo de cereais e massas, leguminosas, leite e derivados, doces e gorduras [12]. Outro estudo, realizado na Ilha de Paquetá/RJ em uma creche com 20 crianças de 2 a 3 anos, observou que os grupos alimentares dos legumes, cereais e do leite não atenderam as recomendações [21].

Neste estudo foram identificadas diferentes formas de alimentação entre as crianças usuárias de um CEI. Apesar da alta ingestão de alimentos industrializados, percebe-se também ingestão de verduras, legumes e frutas com frequência.

Concluiu-se que a frequência de consumo ao dia de açúcar e gorduras, assim como o consumo de suco industrial foi alto, o que merece atenção, pois nem todas as crianças consomem água quando estão em casa. Apesar disso, consumiam verduras, frutas, legumes, e a maioria estava no peso ideal para a idade. Todas as crianças também foram amamentadas, mas poucas até 2 anos, representando um resultado parcialmente satisfatório.

A correta alimentação na infância é muito importante para o adequado crescimento e desenvolvimento da criança, a qual merece atenção por parte dos pais, que são os que ajudam na formação dos hábitos alimentares, através da oferta dos alimentos consumidos pela família. Apesar de toda uma dieta adequada proposta para as crianças no CEI, onde elas passam a maior parte do tempo, em casa os hábitos alimentares da família faz com que a criança consuma alimentos industrializados, que fazem mal a saúde. É necessário o entendimento, por parte dos pais, sobre a importância da alimentação adequada da criança, modificando o comportamento alimentar, não é proibir esses alimentos, mas moderar o consumo.

Este estudo contribuiu para conhecer acerca da alimentação das crianças em casa, o que pode subsidiar intervenções de promoção da saúde junto aos pais. A creche é um espaço de contato entre pais e equipe de saúde que deve ser aproveitado, sendo desnecessária a locomoção dos pais até as Unidades de Saúde. A intersetorialidade com a ligação entre os setores de educação e saúde respeita o princípio de integralidade do Sistema Único de Saúde (SUS) e vai ao encontro dos eixos de ação proposto pelo manual de atenção básica no Brasil, que preconiza o uso das instituições escolares para prática da saúde coletiva. Sugere-se que novos estudos nessa área sejam realizados para investigação das causas dos comportamentos inadequados e avaliação de intervenções para prevenção desses.

Referências

1. São Paulo. Manual de orientação para Centros de Educação Infantil - CEl: esquema alimentar e porcionamentos. 2011. p.52. Disponível em URL: http://portal.sme.prefeitura.sp.gov.br/Portals/1/Files/9370.pdf 
2. Vasconcelos MJ. Nutrição clínica: obstetrícia e pediatria. 1 ed. Rio de Janeiro: Medbook; 2011.740 p.

3. Aires PAP, Souza LCC, Benedetti JF, Blasi CT, Kirsten RV. Consumo de alimentos industrializados em pré-escolares. Rev AMRIGS (Porto Alegre) 2011;55(4):350-5.

4. Ramos M, Stein LM. Desenvolvimento do comportamento alimentar infantil. J Pediatr (Rio J) 2000;76(3):228-37.

5. Ministério da Saúde. Caderneta de saúde da criança: menino/menina. Brasília: Ministério da Saúde; 2011. 92p.

6. Spinelli MGN, Goulart RMM, Santos ALP, Gumiero LC, Farhud CC, Freitas EB et al. Consumo alimentar de crianças de 6 a 18 meses em creches. Rev Nutr 2003;16(4):409-14.

7. Brasil. Ministério da Saúde. Dez passos para uma alimentação saudável: guia alimentar para menores de dois anos. Secretaria de Atenção à Saúde. Departamento de Atenção Básica. $2^{\circ}$ ed. Brasília: Ministério da Saúde; 2013.71p.

8. Brasil. Ministério da Saúde. Secretaria de Atenção à Saúde. Departamento de Atenção Básica. Saúde da Criança: Nutrição infantil: Aleitamento materno e alimentação complementar. Brasília: Ministério da Saúde; 2009.

9. Lacerda CN, Santos SMJ. Aleitamento materno: o conhecimento das mães. Rev Rebes 2013;8(2):9-16.

10. Frota MA, Costa FL, Soares SD, Filho OAS, Albuquerque CM, Casimiro CF. Fatores que interferem no aleitamento materno. Rev Rene 2009;10(3):61-7.

11. Silvia CR, Martins BAET, Oliveira VLMI, Miyaska CK. Consumo alimentar e estado nutricional de pré-escolares de um centro de educação infantil do município de São Paulo. Aliment Nutr 2010;21(3):407-13.

12. Castro TG, Novaes JF, Silvia MR, Brunoro CNM, Franceschini SCC, Tinôco ALA, et al. Caracterização do consumo alimentar, ambiente socioeconômico e estado nutricional de pré-escolares de creches municipais. Rev Nutr 2005;18(3):321-30.

13. Alves M, Muniz LC, Vieira MFA. Consumo alimentar entre crianças brasileiras de dois a cinco anos de idade: Pesquisa Nacional de Demografia e Saúde (PNDS). Ciênc Saúde Coletiva 2013;18(11):3369-77.

14. Toloni MHA, Longo SG, Goulart RMM, Taddei JAAC. Introdução de alimentos industrializados e de alimentos de uso tradicional na dieta de crianças de creches públicas no município de São Paulo. Rev Nutr 2011;24(01):61-70.

15. Brasil. Ministério da Saúde. Secretaria de Atenção Básica. Departamento de Atenção Básica. Obesidade. Brasília: Ministério da Saúde; 2006.

16. Vieira GO, Silvia LR, Vieira TO, Almeida JAG, Cabral VA. Hábitos alimentares de crianças menores de 1 ano amamentadas e não amamentadas. J Pediatr 2004;80(5): 411-6.

17. Barbosa RMS, Salles CR, Soares EA. Guias alimentares para crianças: Aspectos históricos e evolução. Rev Nutr 2006;19(2):255-63.

18. Sociedade Brasileira de Pediatria, Departamento de Nutrologia. Manual de orientação: alimentação do lactante, alimentação do pré-escolar, alimentação do escolar, alimentação do adolescente, alimentação na escola. Rio de Janeiro: Sociedade Brasileira de Pediatria; 2012. 64p.

19. Zaborrskis A, Lagunaite R, Busha R, Lubiene J. Trend in eating habits among Lithuanian school aged children in context of social inequality: three cross-sectional surveys 2002, 2006 and 2010. BMC Public Health 2012;12:52.

20. Philippi ST, Cruz ATR, Colucci ACA. Pirâmide alimentar para crianças de 2 a 3 anos. Rev Nutr 2003;16(1):5-19.

21. Barbosa RMS, Carvalho CGN, Franco VC, Costa RS, Soares EA. Avaliação do consumo alimentar de crianças pertencentes a uma creche filantrópica na llha de Paquetá, Rio de Janeiro, Brasil. Rev Bras de Saúde Materno Infantil 2006;6(1):127-34. 